This item was submitted to Loughborough's Research Repository by the author.

Items in Figshare are protected by copyright, with all rights reserved, unless otherwise indicated.

\title{
The vicinity of an equilibrium three-phase contact line using density functional theory: Density profiles normal to the fluid interface
}

PLEASE CITE THE PUBLISHED VERSION

https://doi.org/10.1080/00268976.2018.1471223

\section{PUBLISHER}

(C) Taylor \& Francis

\section{VERSION}

AM (Accepted Manuscript)

\section{PUBLISHER STATEMENT}

This work is made available according to the conditions of the Creative Commons Attribution-NonCommercialNoDerivatives 4.0 International (CC BY-NC-ND 4.0) licence. Full details of this licence are available at: https://creativecommons.org/licenses/by-nc-nd/4.0/

\section{LICENCE}

CC BY-NC-ND 4.0

\section{REPOSITORY RECORD}

Nold, Andreas, Luis Gonzalez MacDowell, David Sibley, Benjamin D. Goddard, and Serafim Kalliadasis. 2019. "The Vicinity of an Equilibrium Three-phase Contact Line Using Density Functional Theory: Density Profiles Normal to the Fluid Interface". figshare. https://hdl.handle.net/2134/32732. 


\title{
RESEARCH NOTE
}

\section{The vicinity of an equilibrium three-phase contact line using density functional theory: Density profiles normal to the fluid interface}

\author{
Andreas Nold ${ }^{\mathrm{a}, \mathrm{b}}$, Luis González MacDowell ${ }^{\mathrm{c}}$, David N. Sibley ${ }^{\mathrm{d}}$, Benjamin D. \\ Goddard $^{\mathrm{e}}$ and Serafim Kalliadasis ${ }^{\mathrm{b}}$ \\ aTheory of Neural Dynamics Group, Max-Planck-Insititute for Brain Research, 60438 \\ Frankfurt am Main, Germany; ${ }^{\mathrm{b}}$ Department of Chemical Engineering, Imperial College \\ London, London SW7 2AZ, United Kingdom; ' Departamento de Química Física, Facultad de \\ Ciencias Químicas, Universidad Complutense, Madrid 28040, Spain; ${ }^{\mathrm{d}}$ Department of \\ Mathematical Sciences, Loughborough University, Loughborough LE11 3TU, United

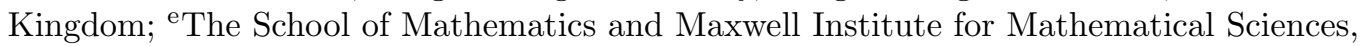 \\ The University of Edinburgh, Edinburgh EH9 3FD, United Kingdom
}

\section{ARTICLE HISTORY}

Compiled April 5, 2018

\begin{abstract}
The paper by Nold et al. [Phys. Fluids, 26, 072001 (2014)] examined density profiles and the micro-scale structure of an equilibrium three-phase (liquid-vapour-solid) contact line in the immediate vicinity of the wall using elements from the statistical mechanics of classical fluids, namely density-functional theory. The present research note, building on the above work, further contributes to our understanding of the micro-scale structure of a contact line by quantifying the strong dependence of the liquid-vapour density profile on the normal distance to the interface, as compared to the dependence on the vertical distance to the substrate. A recent study by Benet et al. [J. Phys. Chem. C, 22079 (2014)] has shown that this could explain the emergence of a film-height dependent surface tension close to the wall, with implications for the Frumkin-Derjaguin theory.
\end{abstract}

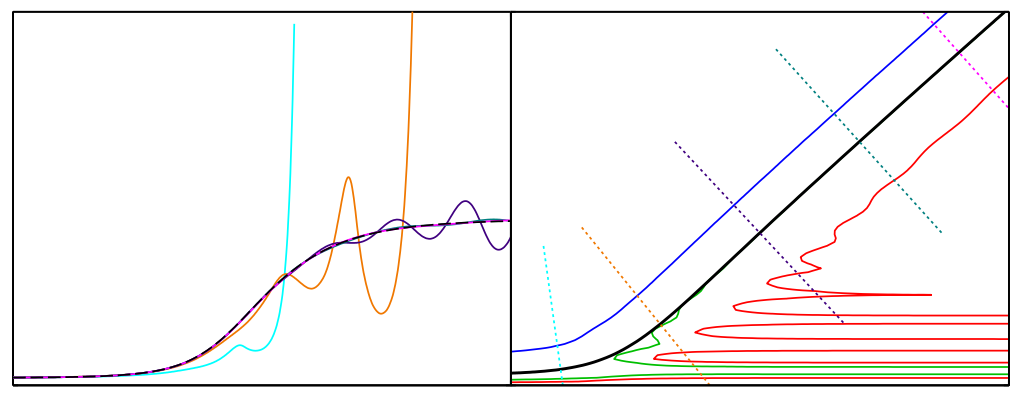

\section{KEYWORDS}

micro-/nanoscale phenomena; non-continuum effects; density-functional theory; contact lines; film-height dependent surface tension

CONTACT Andreas Nold. Email: andreas.nold@brain.mpg.de; Luis González MacDowell. Email: lgmac@quim.ucm.es; David N. Sibley. Email: d.n.sibley@lboro.ac.uk; Benjamin D. Goddard. Email: b.goddard@ed.ac.uk; Serafim Kalliadasis. Email: s.kalliadasis@imperial.ac.uk 


\section{Introduction}

Wetting of solid surfaces has been an active topic of both basic and applied research for several decades due to fundamental questions in the modelling of the inherent multiscale problems associated with the fluid-substrate interaction and because of the numerous applications in diverse fields such as design of nanopatterned superhydrophobic surfaces and surfaces with controllable wetting properties, inkjet printing and the rapidly growing fields of nano-/microfluidics. Relevant recent reviews are given in $[1-3]$.

In recent years, advances in statistical mechanics have allowed greater resolution of the density structure near three-phase contact lines - the location where a vapour, liquid and solid meet. In particular, density-functional theory (DFT) for simple fluids [4] can be used to obtain an accurate picture of the structure at lengthscales commensurate with a single atomic diameter, but at significantly less computational cost than full molecular dynamics (MD) approaches.

Extracting information from MD or DFT simulations to pass parameter values to appropriate coarse-grained models is an active area of research [5-9] and assumptions made should be evaluated in the light of the more accurate nanoscale picture that is now emerging. Quantities of interest are parameters that would enter hydrodynamic descriptions such as Navier-Stokes or reduced thin-film type models, such as surface tensions and viscosities, and then additional functions that arise due to the coarsegraining of the density structure such as binding potentials (or equivalently disjoining pressure).

In this research note, we revisit a crucial assumption of the relatively standard procedure of calculating a binding potential from one-dimensional DFT calculations that are then used in a two-dimensional coarse-grained model with a curved interface. In particular, we contrast the fluid density profile in the vicinity of a contact line normal to the liquid interface, with that for an equilibrium liquid-vapour profile.

In the standard theory of interfaces, the binding potential (expressed as a function of the distance to the solid substrate) is an essential ingredient of the Young-Laplace or Frumkin-Derjaguin equations. However, implicit in such description is the assumption that the density profile everywhere along the film can be parametrized as a simple local function of the film height. On the contrary, it has been recently emphasized that a simple prescription for the density profile as given in terms of normals to the interface does not lead to a strictly local free energy, and provides additional contributions which are at best expressed as corrections to the surface tension [10]. For this reason, it is important to highlight this rather natural distinction between different parametrizations of the density profile.

Section 2 will give a brief background into the computations, and Section 3 will give the new results before conclusions are drawn in Section 4.

\section{Background on computations}

This note naturally builds on the work previously described in Ref. [7] where classical DFT was used to compute the fluid structure in the vicinity of equilibrium contact lines with contact angles $<90^{\circ}$. We briefly describe the fluid model and other relevant details here, but encourage the reader to refer to [7]. for further specific details of the computational methods employed. The case of equilibrium contact $>90^{0}$ degrees are given in Ref. [11] while the pseudospectral numerical method for equilibrium contact 
lines, and wetting problems in general is outlined in Ref. [12]. It is noteworthy that our computational framework is also applicable for some of the most sophisticated freeenergy functionals such as those provided by Fundamental Measure Theory (FMT) by Rosenfeld [13, 14].

Classical DFT is a self-consistent theoretical framework originating from the statistical mechanics of classical fluids. It relates the density structure of a fluid to the minimisation of an appropriate functional $\Omega$, which at its minimum is equivalent to the grand potential. In particular, the Helmholtz free energy $\mathcal{F}$ may be written as a unique functional of the number density profile $n(\mathbf{r})$ at a position $\mathbf{r}[4,15,16]$. To calculate the equilibrium density distribution we numerically solve $\delta \Omega[n] / \delta n(\mathbf{r})=0$, where $\Omega$ is given by

$$
\Omega[n]=\mathcal{F}[n]+\int n(\mathbf{r})\left\{V_{\text {ext }}(\mathbf{r})-\mu\right\} \mathrm{d} \mathbf{r},
$$

with $\mu$ being the chemical potential and $V_{\text {ext }}$ the external potential. We choose an illustrative model of a simple fluid of particles interacting with a Lennard-Jones (LJ) potential by splitting the free energy into a repulsive hard-sphere part and an attractive contribution:

$$
\mathcal{F}[n]=\mathcal{F}_{\mathrm{HS}}[n]+\frac{1}{2} \iint \phi_{\mathrm{attr}}\left(\left|\mathbf{r}-\mathbf{r}^{\prime}\right|\right) n(\mathbf{r}) n\left(\mathbf{r}^{\prime}\right) \mathrm{d} \mathbf{r}^{\prime} \mathrm{d} \mathbf{r},
$$

For the hard-sphere free energy, we employ Rosenfeld's Fundamental Measure Theory (FMT) $[13,14]$. This way, hard-core particle-particle correlations which dominate the liquid structure are treated accurately. For the attractive interactions, we employ a simple van der Waals functional at the level of the Random Phase Approximation (RPA). Unlike FMT, RPA neglects the role of pair correlations in the cohesion energy of the liquid. However, we do not expect this to upset the results at the qualitative level of description that is sought here. For a discussion on possibilities and limitations to include higher order terms, see e.g. [17]. These attractive interactions are modelled with a mean-field Barker-Henderson approach [18], with

$$
\phi_{\text {attr }}(r)=\varepsilon\left\{\begin{array}{ll}
0 & \text { for } r \leq \sigma \\
4\left(\left(\frac{\sigma}{r}\right)^{12}-\left(\frac{\sigma}{r}\right)^{6}\right) & \text { for } r>\sigma
\end{array} .\right.
$$

Here, $\varepsilon$ is the depth of the LJ potential, $\sigma$ is the distance from the center of the particle at which the LJ potential is zero, and $r$ is a (scalar) radial distance.

For the wall-fluid interactions, we similarly use an LJ form which may be appropriately integrated to give

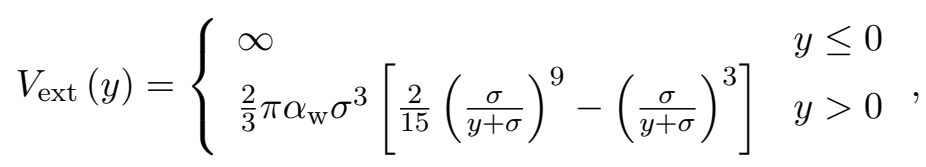

where $\alpha_{\mathrm{w}}$ is the strength of the wall potential and the $y$-coordinate is in the normal direction to the wall.

Computations here are performed at an appropriate temperature of $T=0.75 \varepsilon / k_{B}$, where $k_{B}$ is the Boltzmann constant such that the liquid and vapour phases are dis- 
tinct, and the $2 \mathrm{D}$ computations are performed at the saturation chemical potential.

\section{Results}

In Ref. [7], we studied density profiles of an equilibrium contact line, $n(x, y)$ with $x$, the direction along the substrate and $y$ the outward-pointing direction perpendicular to the substrate. Particular emphasis was given to contact angles $<90^{\circ}$. Using $n(x, y)$, the height of the liquid-vapour interface along the contact line was defined with respect to the Gibbs adsorption through

$$
h(x):=\frac{1}{\Delta n} \int_{0}^{\infty}|n(x, y)-n(x, \infty)| \mathrm{d} y
$$

[which in fact corresponds to the choice $h_{\mathrm{III}}(x)$, Eq. (31) of [7]] with $\Delta n$ the difference between bulk liquid and vapour densities. Note that $h$ as defined in Eq. (5) is a measure for the excess fluid adsorbed on a substrate compared to the bulk density at a given position $x$ along the substrate. Away from the contact line, the film height $h$ and the wall are exactly at the Young contact angle obtained by using interfacial tensions $[7,11,19]$.

In Fig. 3 of a follow-up study [11] specific to contact angles $>90^{\circ}$, density profiles at fixed $x=x_{0}, n\left(x_{0}, y\right)$, were compared with off-saturation equilibrium density profiles, $n_{f}\left(y ; h_{0}\right)$ of planar films of height $h_{0}=h\left(x_{0}\right)$. This gives a good agreement close to the substrate. However, close to the liquid-vapour interface and away from the wall the agreement deteriorates, because the interface is at an angle to the wall and the liquid-vapour interface therefore depends more strongly on the normal distance, $z=z\left(x ; h_{0}\right)$, to the interface than on the vertical distance to the substrate, $y$. A recent study by Benet et al. [10] has shown that this could explain the emergence of a film-height dependent surface tension close to the wall, with implications on the Frumkin-Derjaguin theory (see Section 4).

Here we exploit the previous results for the structure of the contact line $[7,11]$ by plotting the density profiles along cuts perpendicular to the liquid-vapour interface. We do so by performing a change of variables $(x, y) \rightarrow\left(h_{0}, z\right)$, where $h_{0}$ is the height of the liquid-vapour interface and determines the position at which the cut is made, and $z$ is the distance to this position in direction normal to the interface. The positive $z$-direction is always directed towards the substrate (see Fig. 1).

According to our discussion above, the density in the neighbourhood of $z=0$ should compare favourably with the coexistence liquid-vapour density profile $n_{\mathrm{lv}}(z)$, at least for large enough values of $h_{0}$. In Fig. 2, we test when this agreement breaks down as the substrate is approached, for a wide range of contact angles.

We observe that for contact angles less than 90 degrees [subfigures (a) and (b) of Fig. 2], the density profile along the cuts perpendicular to the interface is heavily perturbed by the oscillations of the wall-liquid interface. For cuts made at interfaces less than five molecular diameters (cyan and orange lines), the profile hardly reaches a liquid-like plateau at all. However, already for an interface as close as five molecular diameters away from the substrate, the tail of the profile towards the vapour phase (i.e., negative $z$ ) is very similar to the full liquid-vapour density profile, and gradually develops fully the liquid tail of the profile $(z>0)$ apart from the expected density oscillations that are propagated from the wall due to packing effects. At a distance of approximately 10 particle diameters from the wall, the density profile flattens out and 


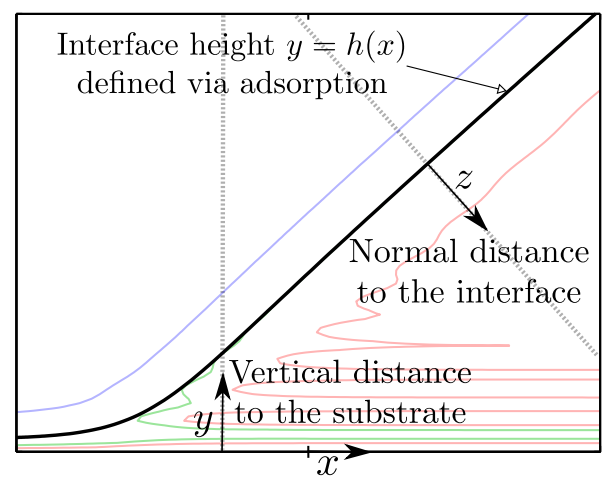

Figure 1. Coordinate system $(x, y)$ located at the substrate, where $y$ is the distance vertical to the substrate, and coordinate system with $\left(h_{0}, z\right)$, where $z$ is the distance normal to the liquid interface. The positive $z$ direction is always directed towards the substrate.

becomes almost indistinguishable from that of a liquid-vapour interface: the interface shape is then not affected by the wall, and it is like having the equilibrium liquidvapour interface there.

For contact angles greater than 90 degrees (subfigures (c) and (d) of Fig. 2), we find that the cuts which fully cross the liquid-vapour interface (cyan and orange lines) closely follow the liquid-vapour profile of a flat interface already for cuts whose interface position is under 5 particle diameters: the packing effects stemming from the wall are not propagated across into the vapour phase and the the agreement of the saturation liquid-vapour interface is very good.

\section{Conclusion}

In this note we have shown that the density profile at a three phase contact line may be described approximately by the saturation density profile of a flat film provided the distance is measured along normals to the interface. One could easily expect this to be the case for interfaces well away from the substrate. Here, we show that for contact angles $>90^{\circ}$ degrees, where the wall-fluid interaction is weaker, this approximation is reasonable down to films with the interface located less than five molecular diameters away. For equilibrium contact angles $<90^{\circ}$, i.e. stronger wall-fluid interactions, the agreement breaks down earlier, approximately 10 molecular diameters away from the substrate. As a note of caution, we highlight that these numbers would depend on chemical heterogeneity and geometrical roughness of the wall, if they were present, as they would impact on the density profile in the vicinity. Also, taking into consideration more complex fluids could affect how quickly the density profile converges to the saturation density profile of a flat film.

The results presented here mean that the effect of the slope of liquid films needs to be taken into account for very thin films in, e.g., capillary theory. This peculiarity seems to have been ignored until recently [10]. To clarify this point, consider the interface potential that results from an external field in the Derjaguin approximation

$$
g(h)=\int V(y) n(y) \mathrm{d} y .
$$

Under the expectation that $g(h)$ should rather be calculated using the density nor- 
(a.I)

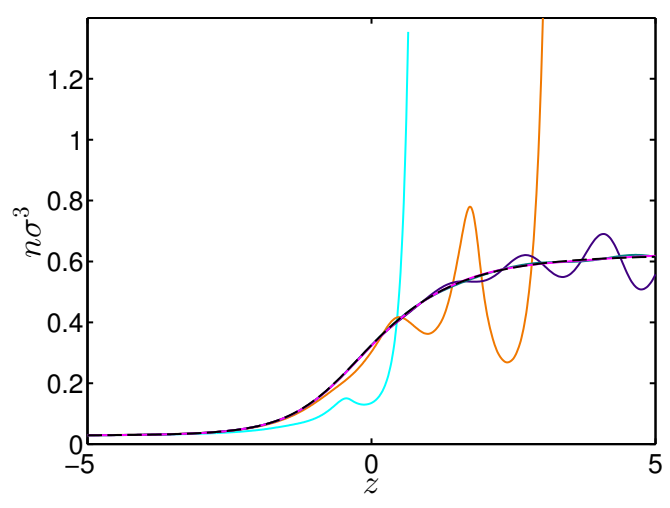

(b.I)

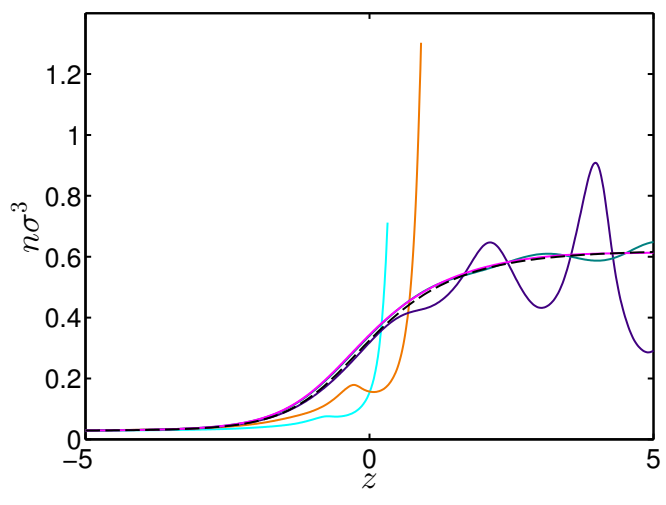

(c.I)

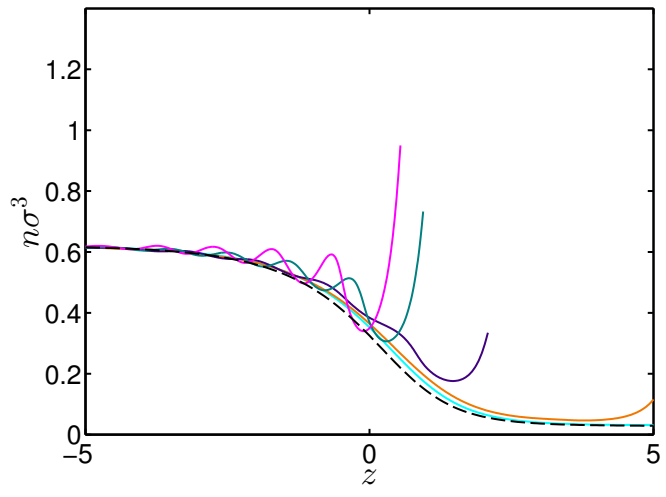

(a.II)

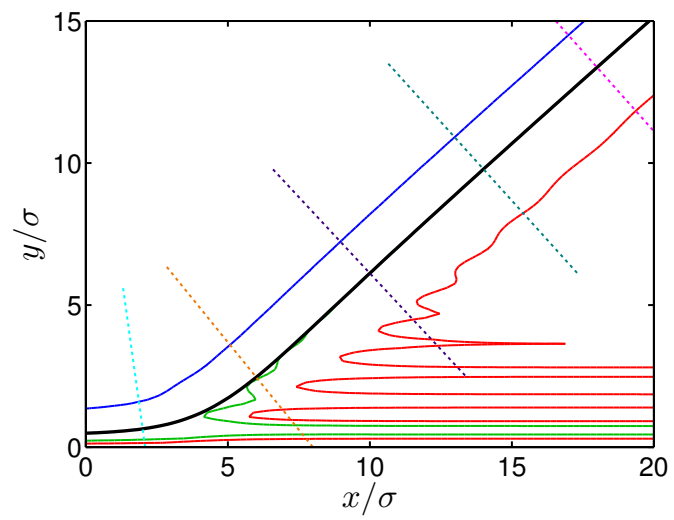

(b.II)

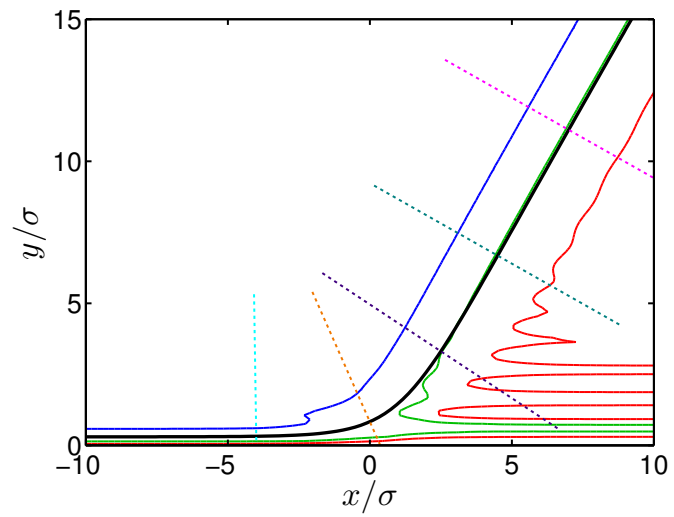

(c.II)

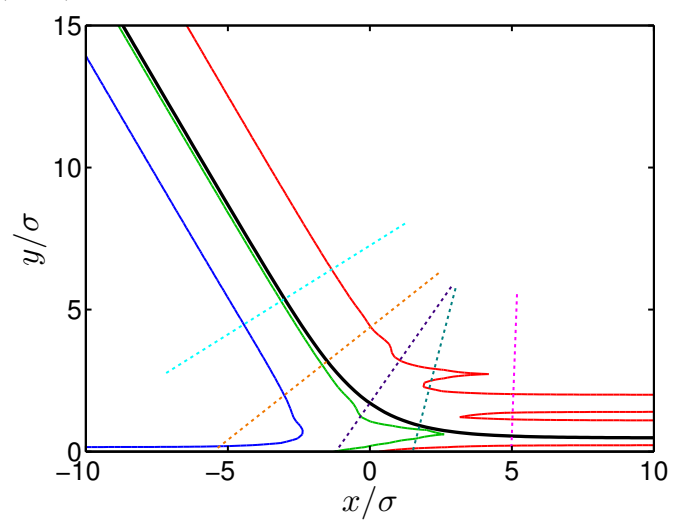


(d.I)

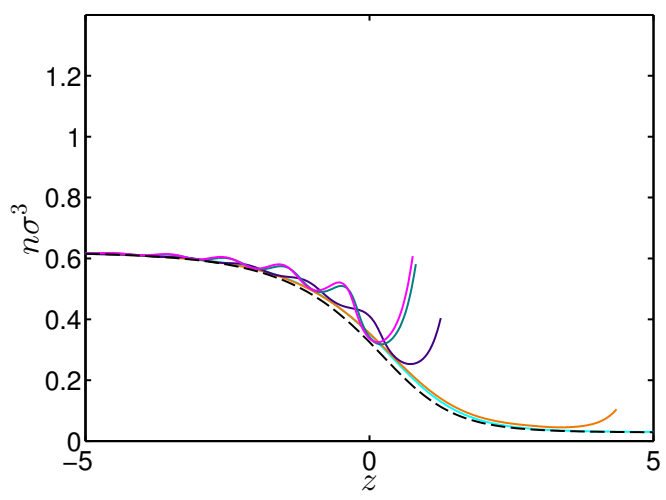

(d.II)

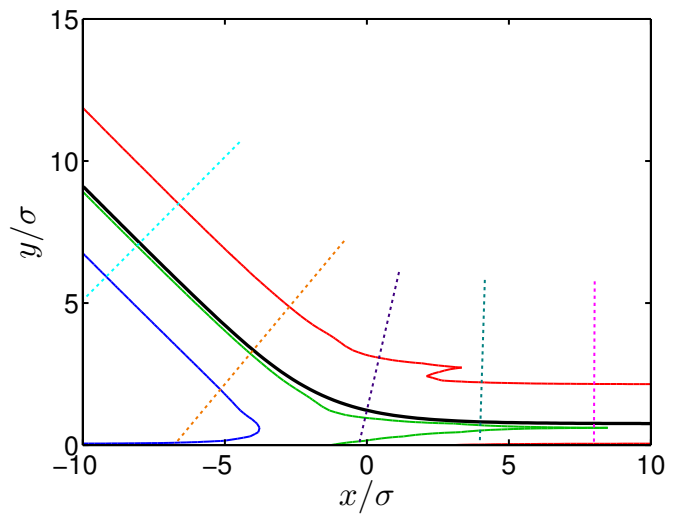

Figure 2. Contour lines of the number density (right column, subfigures II) and density profiles as a function of the distance to the liquid-vapour interface, $z$, at various positions along the interface (left column, subfigures I). In the right column, the blue, green and red contour lines correspond to number densities $\left(n-n_{\mathrm{vap}}\right) /\left(n_{\text {liq }}-n_{\mathrm{vap}}\right)=\{0.05,0.5,0.95\}$ from left to right, respectively. The adsorption height profile defined through equation (5) is depicted by a black solid line. The coloured solid lines in the left column represent the $2 \mathrm{D}$ density profile, plotted along the dashed lines of corresponding color in the right column figures. These density profiles are compared to the liquid-vapour density profile $n_{\mathrm{lv}}(z)$, plotted with a black dashed line in the left column. The strength [7] $\alpha_{w} \sigma^{3} / \varepsilon$ of the substrate for subfigures (a)-(d) is $1.375,1.25,0.7$ and 0.55 , respectively.

mal to the interface i.e. $n(z)$, with $z$ given to a first approximation as $z=(y-$ $h) / \sqrt{1+\left(\partial_{x} h\right)^{2}}$, one finds that $g(h)$ must pick up an additional contribution that is proportional to $\left(\partial_{x} h\right)^{2}$ and depends on the distance from the substrate, whence, effectively, as a film thickness dependent surface tension [10]. The implications of this effect, which could be important for films within the range of the substrate, have not yet been explored.

\section{Acknowledgements}

LGM would like to thank A. Archer and D.N. Sibley for an enjoyable stay at Loughborough University. LGM would also like to acknowledge generous funding by the Spanish Ministerio de Educación, Cultura y Deporte with a travel grant from the Programa Salvador de Madariaga (PRX17/00460). AN, DNS, BDG and SK acknowledge financial support Imperial College (IC) through a DTG International Studentship, from the Engineering and Physical Sciences Research Council (EPSRC) of the UK through Grant No. EP/L027186, EP/L025159 and EP/L020564 and the European Research Council (ERC) through Advanced Grant No. 247031.

\section{Disclosure statement}

No potential conflict of interest was reported by the authors.

\section{References}

[1] D. Quéré, Annual Review of Materials Research 38 (1), 71-99 (2008). 
[2] D. Bonn, J. Eggers, J. Indekeu, J. Meunier and E. Rolley, Rev. Mod. Phys. 81, 739-805 (2009).

[3] J.H. Snoeijer and B. Andreotti, Annual Review of Fluid Mechanics 45 (1), 269-292 (2013).

[4] R. Evans, Adv. Phys. 28 (2), 143 (1979).

[5] L.G. MacDowell, Eur. Phys. J. Special Topics 197 (1), 131-145 (2011).

[6] N. Tretyakov, M. Müller, D. Todorova and U. Thiele, J. Chem. Phys. 138, 064905 (2013).

[7] A. Nold, D.N. Sibley, B.D. Goddard and S. Kalliadasis, Phys. Fluids 26 (7), 072001 (2014).

[8] M. Morciano, M. Fasano, A. Nold, C. Braga, P. Yatsyshin, D.N. Sibley, B.D. Goddard, E. Chiavazzo, P. Asinari and S. Kalliadasis, The Journal of Chemical Physics 146 (24), 244507 (2017).

[9] A.P. Hughes, U. Thiele and A.J. Archer, The Journal of Chemical Physics 146 (6), 064705 (2017).

[10] J. Benet, J.G. Palanco, E. Sanz and L.G. MacDowell, J. Phys. Chem. C 118 (38), 22079 (2014).

[11] A. Nold, D.N. Sibley, B.D. Goddard and S. Kalliadasis, Math. Model. Nat. Phenom. 10 (4), 111 (2015).

[12] A. Nold, B.D. Goddard, P. Yatsyshin, N. Savva and S. Kalliadasis, J. Comput. Phys. 334, 639-664 (2017).

[13] Y. Rosenfeld, Phys. Rev. Lett. 63 (9), 980 (1989).

[14] R. Roth, J. Phys.: Condens. Matter 22 (6), 063102 (2010).

[15] N.D. Mermin, Phys. Rev. 137, A1441-A1443 (1965).

[16] J. Wu, AIChE J. 52 (3), 1169 (2006).

[17] E. Johannessen, J. Gross and D. Bedeaux, J. Chem. Phys. 129 (18), 184703 (2008).

[18] J.A. Barker and D. Henderson, J. Chem. Phys. 47 (11), 4714-4721 (1967).

[19] A. Nold, Ph. D. thesis, Imperial College London, 2016. 